\title{
Preoperative evaluation of peripheral nerve injuries: What is the place for ultrasound?
}

\author{
*Francesca Toia, MD, ${ }^{1}$ Andrea Gagliardo, MD, PhD, ${ }^{2}$ Salvatore D'Arpa, MD, PhD, ${ }^{1}$ \\ Cesare Gagliardo, MD, PhD, ${ }^{3}$ Giuseppe Gagliardo, MD, ${ }^{2}$ and Adriana Cordova, MD ${ }^{1}$ \\ ${ }^{1}$ Plastic and Reconstructive Surgery, Department of Surgical, Oncological, and Oral Sciences, and ${ }^{3}$ Section of Radiological \\ Sciences, Department of Biopathology and Medical Biotechnologies, University of Palermo; and "2Clinical Course" \\ Neurophysiology Unit, Palermo, Italy
}

OBJECTIVE The purpose of this study was to evaluate the usefulness of ultrasound in the preoperative workup of peripheral nerve lesions and illustrate how nerve ultrasonography can be integrated in routine clinical and neurophysiological evaluation and in the management of focal peripheral nerve injuries. The diagnostic role and therapeutic implications of ultrasonography for different neuropathies are described.

METHODS The authors analyzed the use of ultrasound in 119 entrapment, tumoral, posttraumatic, or postsurgical nerve injuries of limbs evaluated in 108 patients during 2013 and 2014. All patients were candidates for surgery, and in all cases the evaluation included clinical examination, electrodiagnostic studies (nerve conduction study and electromyography), and ultrasound nerve study.

Ultrasound was used to explore the nerve fascicular echo-texture, continuity, and surrounding tissues. The maximum cross-sectional area (CSA) and the presence of epineurial hyperechogenicity or intraneural hyper- or hypoechogenicity, of anatomical anomalies, dynamic nerve dislocations, or compressions were recorded.

The concordance rate of neurophysiological and ultrasonographic data was analyzed, classifying ultrasound findings as confirming, contributive, or nonconfirming with respect to electrodiagnostic data. The correlation between maximum nerve CSA and neurophysiological severity degree in entrapment syndromes was statistically analyzed.

RESULTS Ultrasonography confirmed electrodiagnostic findings in $36.1 \%$ of cases and showed a contributive role in the diagnosis and surgical planning in $53.8 \%$ of all cases; the findings were negative ("nonconfirming") in only $10.1 \%$ of the patients. In $16 \%$ of cases, ultrasound was not only contributive, but had a key diagnostic role in the presence of doubtful electrodiagnostic findings. The contributive role differed according to etiology, being higher for tumors (100\%) and for posttraumatic or postsurgical neuropathies (72.2\%) than for entrapment neuropathies (43.8\%).

CONCLUSIONS Ultrasound is a powerful, noninvasive tool for the examination of peripheral nerve injuries, and can guide diagnosis of and surgical strategy for focal peripheral nerve injuries. It allows direct visualization of the cause and extent of nerve lesions and finds its place between electrodiagnostic tests and exploratory surgery. It can provide invaluable information, such as the presence and extent of a mass, scar compression, or neuromas. The authors recommend it as a complement to routine clinical and neurophysiological evaluation and as the first-line imaging modality for masses of suspected nerve origin.

http://thejns.org/doi/abs/10.3171/2015.6.JNS151001

KEY WORDS ultrasound; peripheral nerve; nerve surgery; nerve imaging; electrodiagnosis

$\mathrm{S}$ INCE its first description for the assessment of recurrent laryngeal nerve palsy, ${ }^{30}$ ultrasound nerve imaging has become an established technique for the study of peripheral neuropathies. Several clinical studies have recently demonstrated its high sensitivity and specificity and validated its usefulness in the diagnostic and therapeutic process for focal and generalized peripheral neuropathies. ${ }^{1,4,7,15,19,29}$

High-resolution ultrasound is a reliable tool for examining both the extraneural and the intraneural morphology of peripheral nerves. It allows evaluation of nerve continuity and shape and detection of nerve enlargements, com-

ABBREVIATIONS CMAP = compound muscle action potential; $C S A=$ cross-sectional area; $E M G=$ electromyography; LSD = least significant difference; MUAP = motor unit action potential; SNAP = sensory nerve action potential.

SUBMITTED May 1, 2015. ACCEPTED June 18, 2015.

INCLUDE WHEN CITING Published online January 22, 2016; DOI: 10.3171/2015.6.JNS151001.

* Drs. Toia and A. Gagliardo contributed equally to this work. 
plete or partial lacerations, perineural scars, neuromas and nerve tumors, as well as extrinsic nerve compressions from foreign bodies, neoplasms, implants, or heterotopic ossifications. . $^{1,413,15,19,21}$ Furthermore, ultrasound nerve imaging is simple and fast, readily available, cheap, and causes little discomfort to patients; for these reasons it is a valuable complement to clinical and electrophysiological nerve evaluation, which still represent the gold standard for diagnosis of peripheral neuropathies. ${ }^{11,12}$

Nerve ultrasonography is finding increasing indications for diagnosis and surgical planning of peripheral neuropathies, and due to its advantages over other imaging techniques it has also been proposed as the first-line imaging modality for selected cases. ${ }^{16,33}$

The aim of this study is to retrospectively evaluate our experience with ultrasound in peripheral nerve imaging, to analyze the indications and clinical utility for different neuropathies, and to describe how nerve ultrasonography can be integrated into the routine evaluation and management of focal peripheral nerve injuries.

\section{Methods}

During 2013 and 2014, 119 entrapment, tumoral, posttraumatic, or postsurgical nerve injuries of the limbs were evaluated in 108 candidates for surgery at the authors' institution. The findings and results were retrospectively analyzed. Candidates for surgery were selected based on clinical examination by a surgeon and a neurologist. All of these patients subsequently underwent both electrodiagnostic and ultrasound nerve studies, with the exception of patients in whom electrodiagnostic studies clearly indicated only a radiculopathy, who did not receive ultrasonographic examination. For all patients in this series, preoperative evaluation included clinical examination, electrodiagnostic studies (nerve conduction study and electromyography), and ultrasound nerve study. Neurophysiological and ultrasound examinations was performed in all cases by one of the authors (A.G.) at the "Clinical Course" Neurophysiology Unit in Palermo.

\section{Study Population}

The mean age of the 108 patients was 54.7 years. Most patients with posttraumatic or postsurgical neuropathies were male $(75 \%)$. The most common pathological condition was entrapment neuropathy (69 patients, 80 nerves). The upper limb was involved more frequently than the lower limb (86\% vs $14 \%)$. The mean duration of symptoms was 17.6 months (range 2-72 months); no patient with acute nerve injury was evaluated in this case series. Fifteen percent of the patients had diabetes, and all of these patients presented with entrapment neuropathy (Table 1).

\section{Electrodiagnostic Studies}

Electrodiagnostic examination included motor and sensory conduction velocity studies of the injured nerve and the contralateral homologous and other ipsilateral healthy nerves (data not shown); needle electromyography (EMG) examination of the target muscles was performed according to international guidelines. ${ }^{3,6,26,31}$ Sensory nerve action potentials (SNAPs) and compound muscle action poten- tials (CMAPs) were classified as normal, pathological, or absent; pathological response included a reduction of the motor or sensory conduction velocity values or a reduction of the SNAP or CMAP voltage. Eventual denervation potentials and motor unit action potential (MUAP) recruitment patterns (interference, intermediate, single MUAPs, absent) were recorded by EMG. A NeMus 2 EBNeuro (Florence, Italy) neurophysiological equipment was used.

\section{Ultrasonography}

Ultrasound scanning was performed on the injured nerve when the clinical diagnosis was confirmed by neurophysiological examination; in cases of doubtful or confounding neurophysiological findings, all main nerves of the same limb were explored. Each nerve was visualized along its course throughout the whole limb, exploring the nerve fascicular echotexture, continuity, and surrounding tissues; the presence of epineurial hyperechogenicity or intraneural hyper- or hypoechogenicity was recorded. The maximum cross-sectional area (CSA) was measured at the inner border of the epineurium. For posttraumatic and postsurgical cases-which involved different nerves at different levels-CSA values were normalized as a percentage of the contralateral healthy nerve, which was used as reference to facilitate analysis of the results. For entrapment syndromes - which are often bilateral-CSA was expressed in square millimeters $\left(\mathrm{mm}^{2}\right)$ and compared with reference values form the literature. ${ }^{25}$ For tumors, CSA was also expressed in square millimeters. Color Doppler ultrasound scans were performed for suspected tumors.

The presence of anatomical anomalies, dynamic nerve dislocations, or compressions was recorded. An Esaote MyLab 25 Gold ultrasound system equipped with a broadband (frequency band 10-18 MHz) linear transducer was used.

\section{Data Analysis}

Electrodiagnostic and ultrasonographic data were grouped according to etiological diagnosis into entrapment neuropathies, posttraumatic or postsurgical neuropathies, and tumors, and intra- and intergroup analyses were performed. Posttraumatic or postsurgical neuropathies were further classified as neuromas, nerve compressions, and traction neuropathies, according to clinical history and final clinical and neurophysiological/ultrasonographic diagnosis.

The concordance rate of neurophysiological and ultrasonographic data was analyzed for each group. Ultrasonographic findings were correlated to the electrodiagnostic findings to evaluate their role in diagnosis and development of surgical strategy; results were classified as contributive (influenced diagnostic and therapeutic strategies), confirming (confirmed clinical and neurophysiological diagnosis), nonconfirming (normal ultrasound findings), or incorrect (led to incorrect diagnosis), according to Padua's evaluation scale. ${ }^{25}$

\section{Statistical Analysis}

Statistical analysis was performed using IBM SPSS Statistics, version 20.0 (IBM Corp.). Univariate analysis of 
TABLE 1. Characteristics of the study population*

\begin{tabular}{|c|c|c|c|c|c|c|c|c|c|}
\hline \multirow[b]{2}{*}{ Pathological Condition } & \multirow{2}{*}{$\begin{array}{l}\text { No. of } \\
\text { Patients }\end{array}$} & \multirow{2}{*}{$\begin{array}{l}\text { No. of } \\
\text { Nerves }\end{array}$} & \multirow{2}{*}{$\begin{array}{l}\text { Mean Age } \\
\quad(y r s)\end{array}$} & \multicolumn{2}{|c|}{ Sex } & \multicolumn{2}{|c|}{ Limb } & \multirow{2}{*}{$\begin{array}{l}\text { Mean Duration of } \\
\text { Sx (mos) }\end{array}$} & \multirow[b]{2}{*}{ Diabetes } \\
\hline & & & & $M$ & $\mathrm{~F}$ & Upper & Lower & & \\
\hline Entrapment neuropathies & 69 & 80 & $56.7 \pm 15.6$ & $33(48)$ & $36(52)$ & $76(95)$ & $4(5)$ & $18.2 \pm 19.7$ & $16(23)$ \\
\hline $\begin{array}{l}\text { Posttraumatic or postsurgical } \\
\text { neuropathies }\end{array}$ & 36 & 36 & $50.2 \pm 19.2$ & $27(75)$ & $9(25)$ & $24(67)$ & $12(33)$ & $17.2 \pm 20.2$ & 0 \\
\hline Tumors & 3 & 3 & $63.3 \pm 5.7$ & $0(0)$ & $3(100)$ & $2(67)$ & $1(33)$ & $12 \pm 3.4$ & 0 \\
\hline Total & 108 & 119 & $54.7 \pm 17.2$ & $60(56)$ & $48(44)$ & $102(86)$ & $17(14)$ & $17.6 \pm 19.5$ & $16(15)$ \\
\hline
\end{tabular}

Sx = symptoms.

* Values represent numbers of patients or lesions (\%) unless otherwise indicated. Mean values are presented with SDs.

variance (ANOVA) was performed for carpal tunnel syndrome, using the maximum nerve CSA as the dependent variable and neurophysiological severity classification as the independent variable; a post hoc Fisher's least significant difference (LSD) analysis was performed. An independent-samples t-test was performed for cubital tunnel syndrome, using maximum nerve CSA as the dependent variable and neurophysiological severity classification as the independent variable. A $p$ value $\leq 0.05$ was regarded as significant.

\section{Results}

Electrodiagnostic and ultrasonographic findings for the 3 different groups are reported in Tables 2 and 3, and their analysis is reported below.

\section{Entrapment Neuropathies}

Carpal tunnel and cubital tunnel syndromes were classified according to the severity of electrodiagnostic alterations. $3,26,31$

In carpal tunnel syndrome, the mean maximum CSA of the median nerve at the wrist significantly increased with the severity of these alterations (ANOVA, $p=0.001$; $\mathrm{F}=8.866$ ), ranging from a mean of $9 \mathrm{~mm}$ in the minimal/ mild subgroup-which falls in the range of normal values $^{25}$ - to a mean of 20 in the severe/extreme subgroup. Further, a post hoc Fisher's LSD analysis for intergroup analysis showed a significant difference of CSA values between the mild vs moderate $(\mathrm{p}=0.02)$, moderate vs severe $(p=0.01)$ and severe vs mild group $(p=0.001)$. The presence of epineurial hyperechogenicity also increased with the severity of electrodiagnostic alterations, and was present in $83.3 \%$ of patients with extreme neurophysiological alterations and $25 \%$ of those with severe alterations, while it was not found in minimal and mild cases. Nerve hypoechogenicity was never observed in the minimal/ mild subgroup, but was always observed in the moderate and severe/extreme subgroups. Also, ultrasound showed anatomical variations predisposing to the entrapment syndrome, namely a bifid median nerve and/or a median artery in 8 patients (17.4\%) (Fig. 1).

In cubital tunnel syndrome, the mean CSA of the ulnar nerve at the elbow did not differ significantly between the mild and moderate subgroups (t-test for independent variables), but mean CSA values were always increased relative to normative data in the literature, ${ }^{25}$ also in mild cases. Epineurial hyperechogenicity was observed in $40 \%$ of mild cases and $100 \%$ of moderate cases. Nerve hypoechogenicity was observed in all cases, irrespectively of their severity. Also, ultrasound showed nerve dislocation at the elbow in $14.8 \%$ of patients.

In the patient with Guyon's canal syndrome, the electrodiagnostic findings were pathological; ultrasound showed a normal nerve CSA $\left(4.9 \mathrm{~mm}^{2}\right)$ but revealed the cause of the nerve entrapment, namely a ganglion cyst, guiding the planning of surgical treatment (Fig. 2).

Also, ultrasound complemented the electrodiagnostic diagnosis of anterior interosseous nerve and posterior interosseous nerve entrapments by localizing the site of compression, indicated by an increase of nerve CSA proximally to it (mean values 3 and $4 \mathrm{~mm}^{2}$, respectively) compared with the healthy contralateral side, and by nerve hypoechogenicity.

Data on the lower limb are limited by the small sample size. In common peroneal nerve entrapment, increased nerve CSA (mean $30 \mathrm{~mm}^{2}$ ) and epineurial hyperechogenicity accompanied the pathological electrodiagnostic findings, while in a case of lateral femoral cutaneous nerve entrapment, the only study that confirmed the suspected clinical diagnosis was ultrasonography, which demonstrated a maximum nerve CSA of $6 \mathrm{~mm}$.

\section{Posttraumatic and Postsurgical Neuropathies}

Posttraumatic or postsurgical cases were characterized by a great variability of the type and level of injury.

Neuromas always showed a greatly increased CSA (mean $257.8 \% \pm 67.4 \%$ ), while routine electrodiagnostic findings were normal in $22.2 \%$ of cases and pathological in $77.8 \%$ (with $22.2 \%$ of cases showing a complete axonotmetic/neurotmetic pattern). In all cases, the nerve was hypoechoic, but most importantly, it showed an altered echotexture, diagnostic of neuroma (Fig. 3). Also, in all cases of neuroma, ultrasound allowed measurement of the longitudinal and axial size of the lesion, and a Tinel sign was elicited at the passage of the probe, confirming the diagnosis and localization. The possibility of moving the probe in different planes allows visualization of the whole nerve and assessment of the type of damage (i.e., partial or complete involvement) (Fig. 4).

In the nerve compression subgroup, ultrasound showed an increased CSA (mean $171.3 \% \pm 67.2$ ), a frequent nerve hypoechogenicity (66.7), and an epineurial hyperechogenity in $38.9 \%$ of cases; also, multifocal damage, characterized by doubtful, nonconclusive, electrodiagnostic find- 


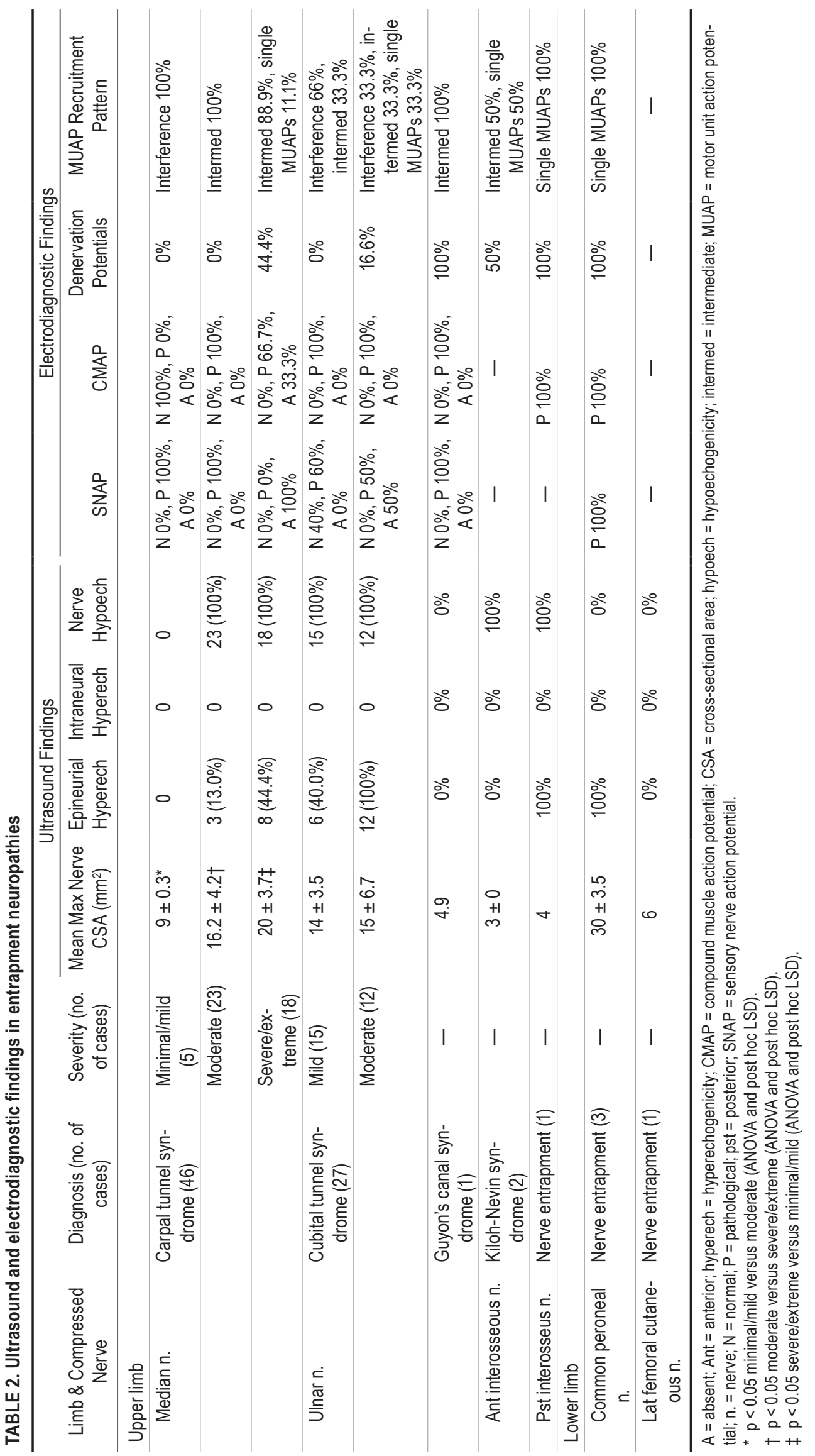




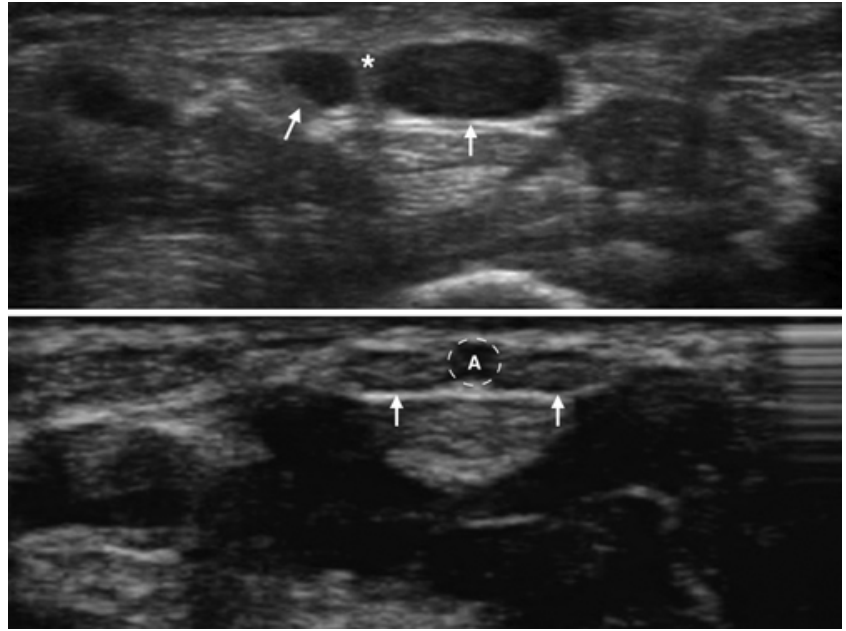

FIG. 1. Ultrasound axial scan of the median nerve at the wrist, showing 2 anatomical variants associated with carpal tunnel syndrome. Upper: Bifid median nerve in axial scan at the wrist. The nerve is divided by a fibrous hyperechoic septum (asterisk) into 2 compartments (arrows). Lower: A persistent median artery $(\mathrm{A})$ can be found within this septum.

ings, was identified in $22.2 \%$ of cases. Ultrasound also revealed morphological details that guided not only the etiologic diagnosis, but also surgical strategy, such as the presence of perineural scarring, heterotopic ossification (Fig. 5), or foreign bodies (Fig. 6).

In the traction neuropathy subgroup, all patients showed pathological electrodiagnostic findings. Ultrasound showed an increased CSA (mean 137.3\% $\pm 67.1 \%$ ), an epineurial hyperechogenicity in almost half of the cases (44.4\%), and an alteration of intraneural echogenicity in $66.7 \%$ of cases (hyperechogenicity in $33.3 \%$, hypoechogenicity in $33.3 \%$ ). Individual analysis of CSA and nerve echogenicity guided diagnosis and surgical strategy (Fig. 7).

\section{Tumors}

In the 3 cases of schwannoma, routine electrodiagnostic findings were always normal, and diagnosis was achieved with ultrasound, which also provided information on the size and the vascular supply of the tumor (Figs. 8-10, Video 1).

VIDEO 1. Video clip of a dynamic ultrasound axial scan of the median nerve along the arm. Moving the probe along the limb, maintaining the nerve in the center of the screen, long tracts of nerves are easily and quickly explored. In this case, details on which nerve and on how many fascicles are intact could also be achieved (also see Figs. 8-10). Copyright Andrea Gagliardo. Published with permission. Click here to view.

The CSA was greatly increased (mean $105 \pm 84.3$ $\mathrm{mm}^{2}$ ), and in 1 case an intratumoral calcification was identified. In all 3 cases, diagnosis was confirmed by subsequent MRI.

\section{Role of Ultrasound}

Ultrasound confirmed electrodiagnostic findings in $36.1 \%$ of the patients of this case series (47.5\% of entrapment neuropathies and $13.9 \%$ of posttraumatic or postsur- 


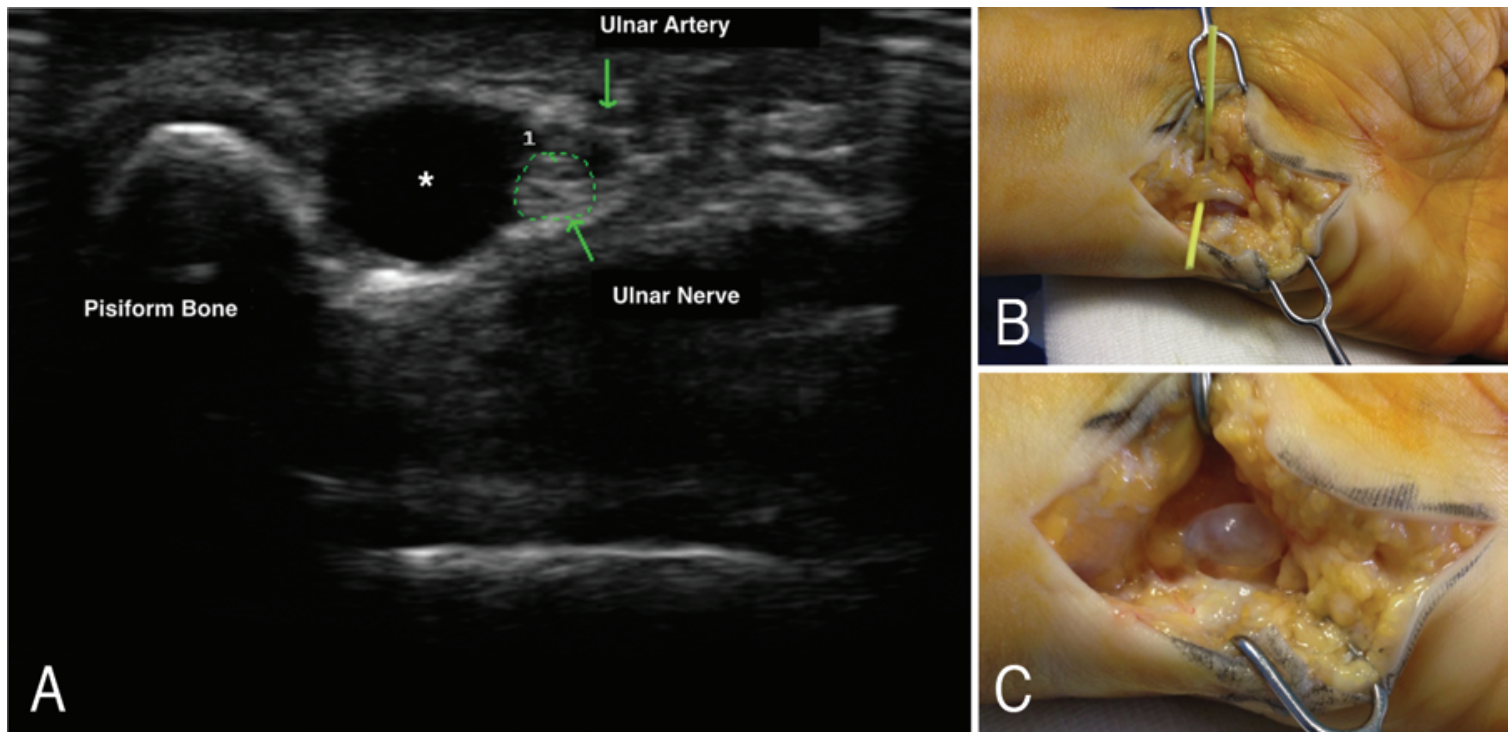

FIG. 2. A: Ultrasound axial scan of the Guyon canal at the wrist. The ulnar nerve is generally located between the pisiform bone and the ulnar artery. In this case, ultrasound revealed a ganglion cyst (asterisk) occupying the canal and causing nerve compression. B: Intraoperative photograph obtained after nerve exposure and decompression but before removal of the ganglion cyst. C: Intraoperative photograph showing the cyst. The presence of the cyst was known beforehand and the risk of missing it was avoided. Figure is available in color online only.

gical neuropathies), and showed a contributive role in diagnosis and surgical strategy in $53.8 \%$ of cases. This rate was higher for tumors $(100 \%)$ and posttraumatic or postsurgical neuropathies (72.2\%) (Fig. 11). In $16 \%$ of cases, ultrasound was not only contributive, but had a key diagnostic role in the presence of doubtful electrodiagnostic findings.

Ultrasound showed a contributive role in $43.8 \%$ of the cases of entrapment syndrome, which included the identification of a concurrent flexor tenosynovitis (in $8.8 \%$ of cases), perineural scar (3.8\%), or anatomical variations, such as a bifid median nerve and/or a median artery (10\%),
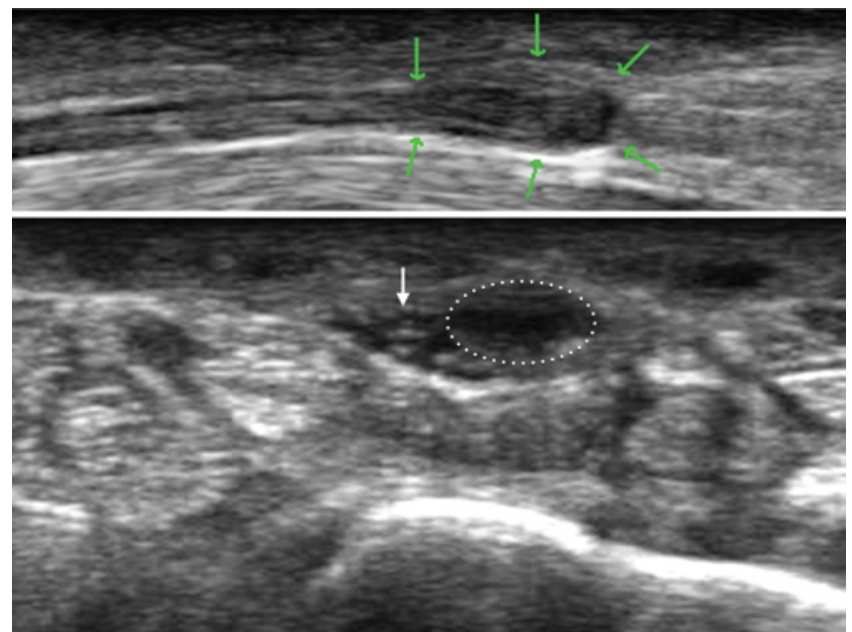

FIG. 3. Ultrasound scans of the forearm showing the typical shape of a neuroma in longitudinal (upper) and axial (lower) views of the median nerve. The axial scan shows a partial neurotmesis of the nerve with preservation of a significant percentage of nerve fascicles (arrow) in continuity below the neuroma. Figure is available in color online only. and nerve dislocation at the elbow in $11.3 \%$ of cases. Also, ultrasound contributed by the identifying a compressive mass in the Guyon canal (1.3\%) and uncommon sites of compression $(5 \%)$ or dynamic compressions $(2.6 \%)$ not identified by electrodiagnostic findings. Lastly, it contributed to the diagnosis of Parsonage-Turner syndrome in a patient with ulnar nerve palsy, by excluding the presence of focal lesions along the whole limb (1.3\%).

Ultrasound had a contributive role in $72.2 \%$ of traumatic cases, mainly due to identification of nerve continuity. It identified the presence and extension of both terminal neuromas and neuromas-in-continuity in 25\% of cases $(100 \%$ of suspected neuromas). In compression and traction neuropathies, ultrasound allowed a direct visualization of nerve continuity and eventual surrounding hyperechoic (fibrous) tissue that contributed to the neuropathy, leading to diagnosis in $13.9 \%$ of posttraumatic/postsurgical cases. For instance, a suspected ulnar nerve injury in a patient who had sustained an elbow fracture was diagnosed as a brachial plexopathy, while in 2 postsurgical cases of fibula fracture, a concurrent scar neuropathy of the superficial peroneal nerve (in 1 case) or of the common and superficial peroneal nerves (in 1 case) was identified. In compression neuropathies, ultrasound also allowed the distinguishing of different anatomical pictures in patients with similar clinical and electrodiagnostic findings, contributing to diagnosis and surgical planning in $11.1 \%$ of cases. For instance, in 2 extreme cases in which sensory and motor ulnar potentials could not be evoked subsequent to fractures, ultrasound showed a greatly increased nerve CSA at the elbow without other significant findings in 1 case, and excessive bone deposition (Fig. 5) that surrounded and compressed the nerve in the second case. In another case, ultrasound showed a mild reduction of the space within the condylar groove, resulting in a superficial position of the ulnar nerve 

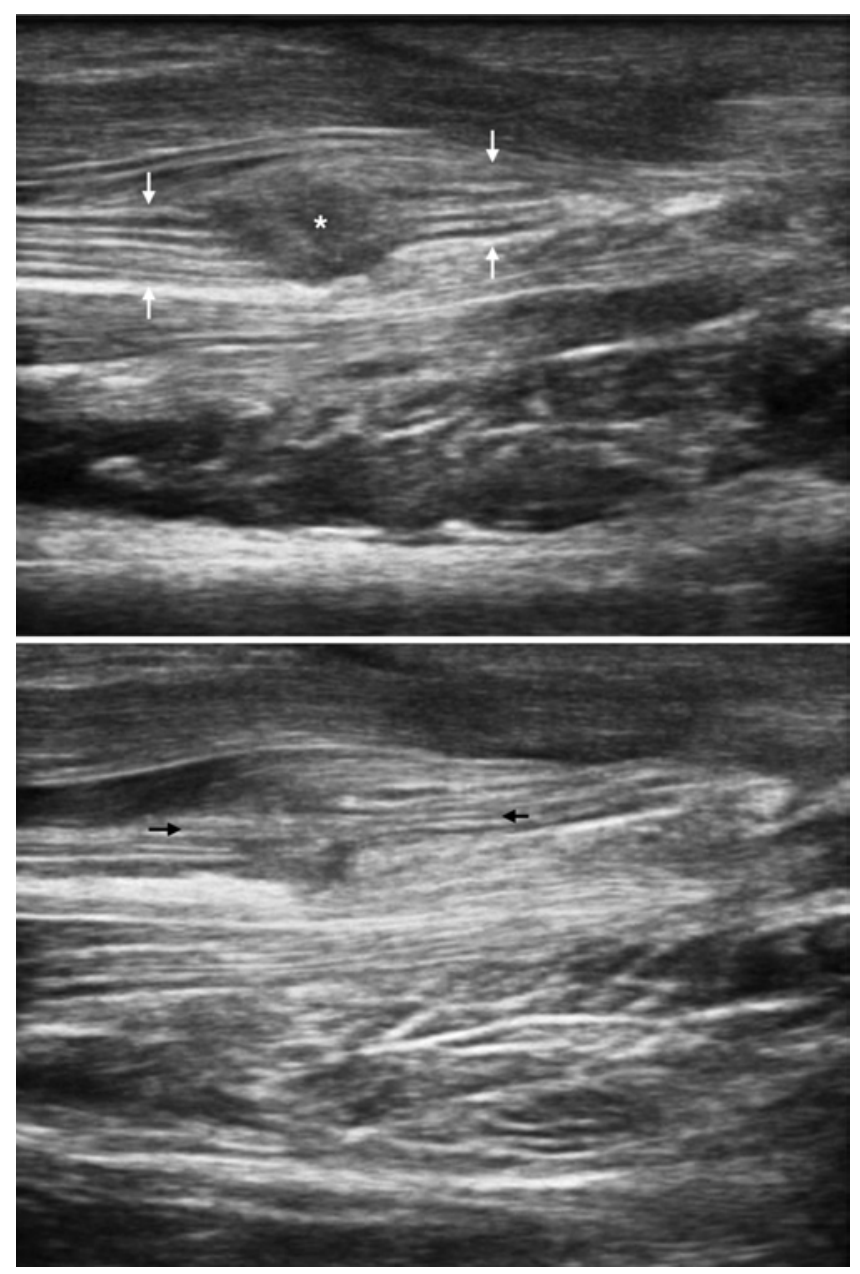

FIG. 4. Longitudinal ultrasound scans of the median nerve. Note the interruption of many fascicles (white arrows) for the presence of a neuroma (asterisk). Slightly moving the probe, different planes of the nerve can be explored and a small number of fascicles (black arrows) show their continuity across the lesion. These are the advantages of a dynamic real-time high-resolution ultrasound examination.

and dynamic subluxation. Ultrasound also identified multiple sites of injury caused by the same trauma and not immediately recognized by neurophysiological examination or helped diagnosis in patients with a previous nerve injury before trauma in $22.2 \%$ of cases; for instance, 2 patients with a brachial plexopathy had a concurrent ulnar or radial nerve injury, and a patient with a history of hip surgery and L-5 radiculopathy had a challenging common peroneal nerve injury after a mild knee sprain.

Ultrasound had a contributive role in $100 \%$ of tumor cases, allowing visualization and providing anatomical details of the lesions.

The results of ultrasound examination were negative ("nonconfirming" with respect to electrodiagnostic findings) in only $10.1 \%$ of cases-all minimal-grade carpal tunnel syndromes, 1 median nerve posttraumatic compression, 1 common peroneal nerve traction injury, and 2 mild cases of brachial plexus traction neuropathy. The results of ultrasound examination were also negative in 1 case of mild cubital tunnel syndrome, 1 case of suspected

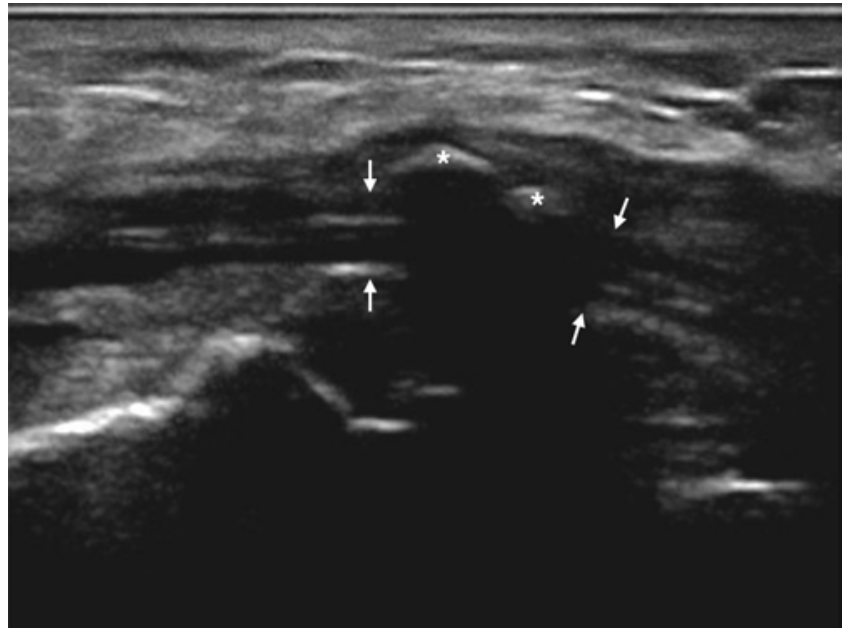

FIG. 5. Ulnar nerve (arrows) in longitudinal ultrasound scan at the elbow. After a displaced fracture of the elbow, the patient did not recover any motor or sensory ulnar function. Ultrasound showed deposits of bone (asterisk) over the nerve fascicles, as a "fatal embrace" with the ulnar nerve. Note the shadow cone of the ultrasound that does not pass through the bone and obscures the underlying nerve.

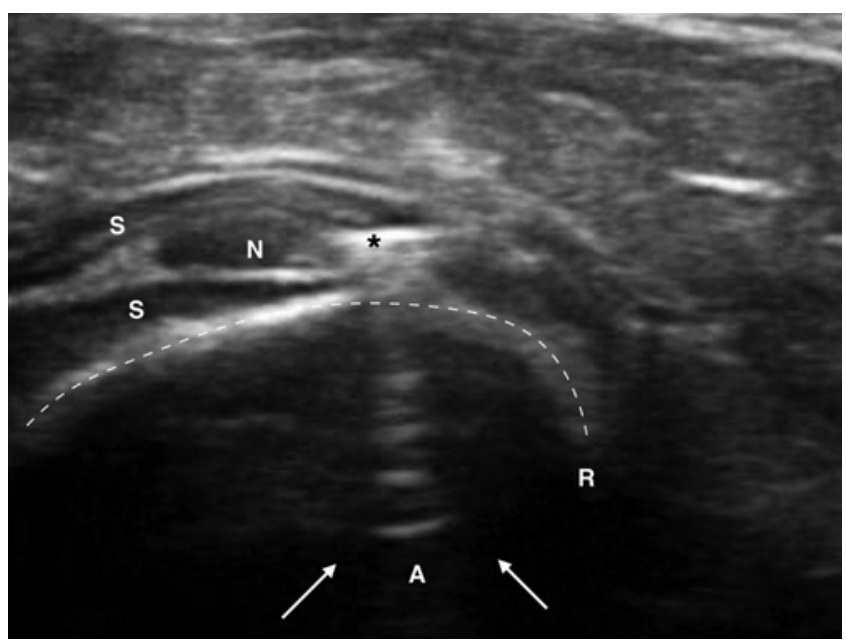

FIG. 6. Ultrasound axial scan of the posterior interosseus nerve $(\mathrm{N})$ between the superficial and deep heads of the supinator muscle (S) in a patient who had suffered a hunting accident. The nerve has a focal huge increase of its CSA. A small hyperechoic metallic bullet (asterisk), easy to identify because of the prominent ultrasound artifact that it generates (arrows), pushes laterally and compresses the nerve, causing a deficit of the finger extension. The bullet is located on the superficial profile of the cortical bone of the radius $(R)$. With an exact preoperative diagnosis surgery can be targeted without the need for wide exploration and dissection.

ulnar nerve entrapment that resulted in a diagnosis of cervical myeloradiculopathy, and 1 case of foot drop due to partial proximal sciatic nerve impairment.

\section{Discussion}

The purpose of this study was to evaluate the usefulness of ultrasound in the preoperative workup of peripheral nerve lesions. In our clinical series, routine ultrasound 


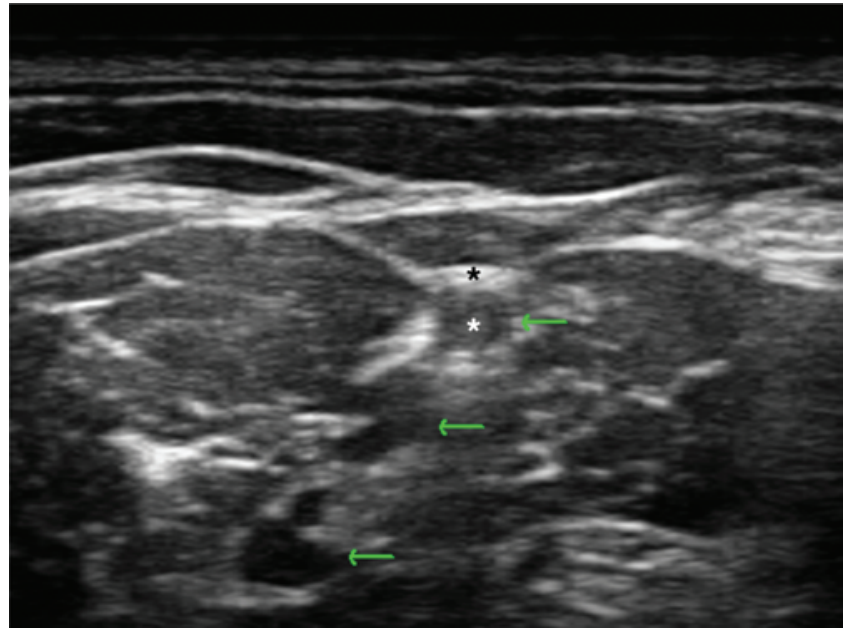

FIG. 7. Superior trunk brachial plexopathy. Ventral branches of the C-5 (upper arrow), C-6 (middle arrow), and C-7 (lower arrow) spinal nerves in the interscalenic area. Note that the superior trunk shows an inner hyperechogenicity (white asterisk) and is surrounded by a thick hyperechoic fibrous tissue (black asterisk). This pattern suggests a stretching injury of the nerve trunk. Figure is available in color online only.

assessment was always added to standard clinical and neurophysiological examination of patients with peripheral neuropathies who were candidates for surgery and proved useful both as a diagnostic tool and in surgical planning. Our decision to introduce ultrasound as a routine examination for surgical candidates was driven by the expectation of clinical utility: an easier surgery in entrapment injuries due to the anatomical details provided and a lower need for MRI in posttraumatic/postsurgical injuries.

Ultrasound allows real-time evaluation of lesions. Within our team, there is a close cooperation between the surgeons, the neurophysiologist, and the neuroradiologist: this means that unexpected findings can be immediately discussed and further assessment immediately performed based on the surgeon's indications, thus tailoring the examination to each patient to provide accurate information useful for surgical treatment.

The diagnostic contribution of ultrasound was higher for focal masses arising from or compressing the nerve, such as nerve tumors, neuromas, foreign bodies or ganglion cysts, and in complex posttraumatic or postsurgical neuropathies; in our series it allowed diagnosis in $16 \%$ of cases with discordant clinical and electrodiagnostic findings. Its practical contribution in the planning of the surgical access and strategy was even higher; besides being the reference instrumental evaluation in the above-mentioned cases, it seems to contribute to a faster and easier surgery for entrapment neuropathies. Thanks to the anatomical details provided, the surgeon knows in advance about anatomical anomalies or dynamic nerve dislocations; also, we hypothesize that the presence of hyperechoic fibrous tissue could indicate epineurial fibrosis and the need for neurolysis. Surgeons perceived these data as facilitating and speeding surgery; however, we did not measure surgical times or correlate ultrasonographic and surgical findings, which we are currently investigating in a prospective study.
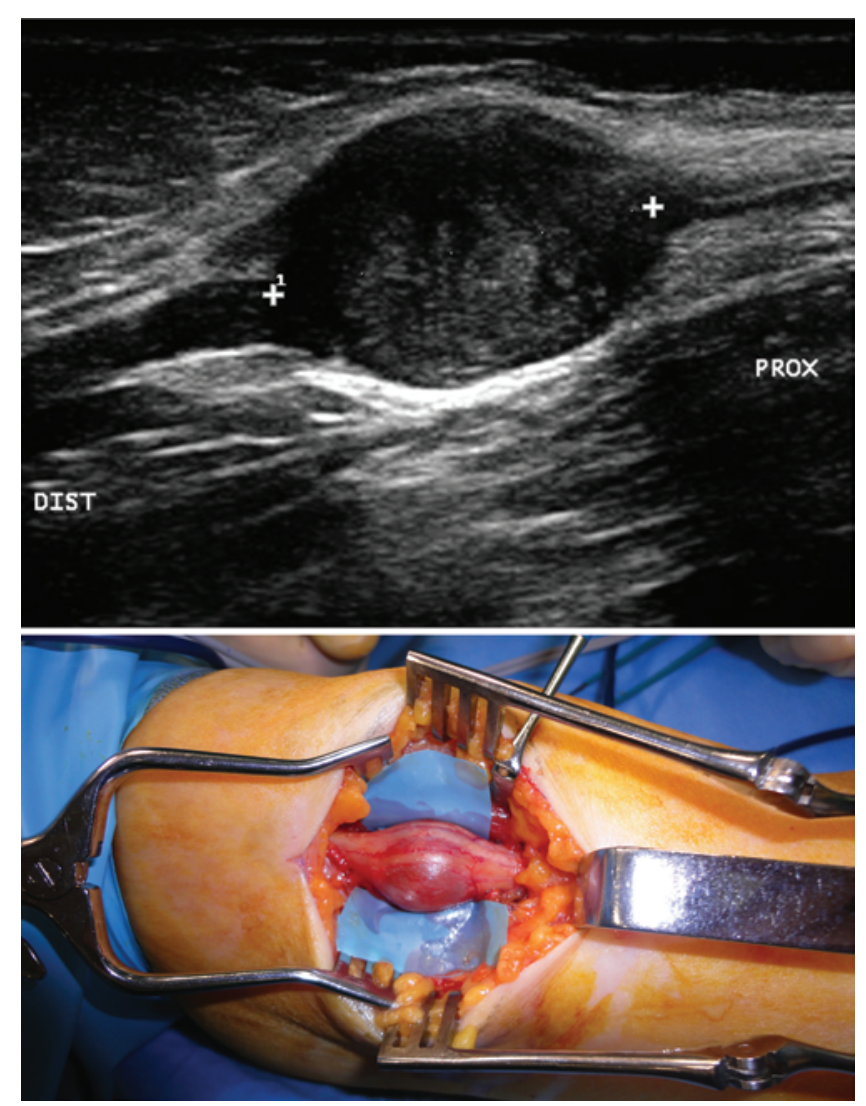

FIG. 8. Upper: Schwannoma of the median nerve at the mid-arm in a longitudinal ultrasound scan. Following the median nerve by ultrasound, from the distal carpal tunnel to the axilla, we clearly show a mass, which originates from within this nerve and which displaces and does not infiltrate most of nerve fascicles. Lower: Intraoperative photograph showing the lesion. The diagnosis of schwannoma was confirmed by surgical exploration and histological examination. This case beautifully illustrates how close the ultrasound image is to reality. In this particular case, already conscious of the diagnosis and knowing that the nerve conduction and continuity are intact, thanks to the unparalleled high-resolution details, ultrasound imaging allowed for improvements in patient information, surgical planning, and decision making. Figure is available in color online only.

Ultrasound is a reliable, cheap, and readily available diagnostic tool. Its accuracy is similar to that of MRI. Although MRI is generally recognized as having a better contrast, and is still preferable for the study of deep nerves, ultrasound has been reported to have a greater sensitivity and an equal specificity, and to allow a better identification of multifocal lesions for peripheral nerve lesions. ${ }^{33}$ MRI for nerve visualization is better performed in preselected regions and by high magnetic field scanners (1.5 T or higher). Although T1-weighted spin-echo sequences offer good morphological evaluation, and the "MR neurography" technique ${ }^{10,18}$ provides a high-contrast nerve signal by the use of highly weighted T2 sequences with long echo times for muscle suppression, selective fat suppression, and spatial radiofrequency pulses outside the imaging volume for vessel suppression, the presence of some metallic devices could contraindicate or severely hamper the MR image acquisition. 

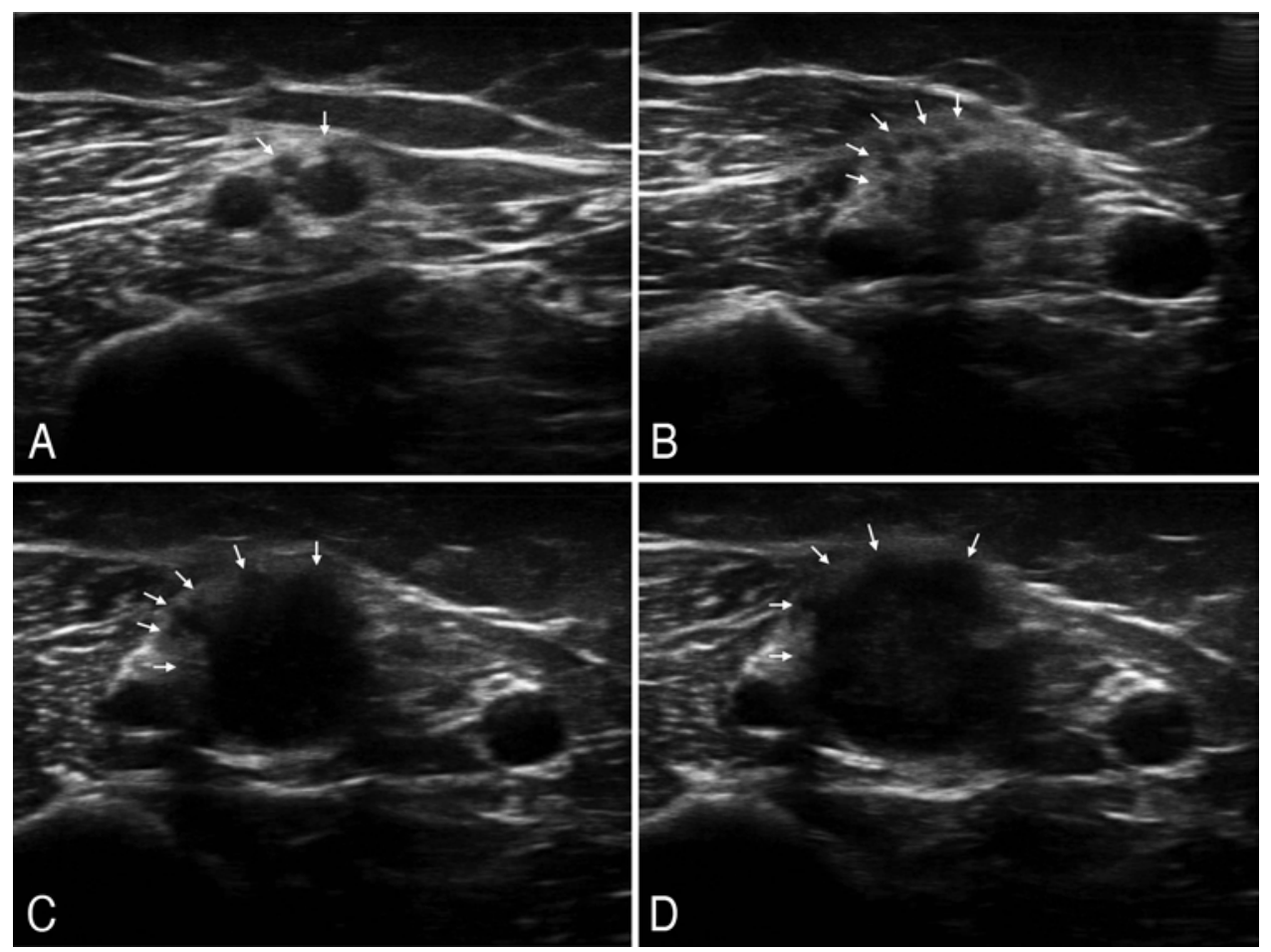

FIG. 9. A-D: Axial ultrasound images showing the same schwannoma as in Fig. 8. Schwannomas usually displace most axon fibers, which can be spared during tumor enucleation. In axial live scans, the fascicles surrounding the tumor could be followed along the nerve (arrows) (also see Video 1).

Ultrasound has no absolute contraindications in common practice, and its advantages over MRI include being less time-consuming and allowing real-time, dynamic, and serial examination of a wide anatomical field as well as visualization of a long nerve in a single scan. ${ }^{17}$ Also, it can easily assess flow dynamics and vascular patterns, which provide further anatomical details that can be especially useful in the diagnosis of nerve tumors (Fig. 10). Furthermore, ultrasonography can be performed at the same time as the clinical examination and electrodiagnostic study, allowing a complete morphological and functional examination of the nerve.

Based on these findings, we believe that ultrasound is likely to replace MRI as the first-line imaging technique for focal lesions arising from or compressing a nerve; however, a limited number of cases were analyzed in this series, and this assumption need validation on a larger scale.

\section{Entrapment Neuropathies}

Our results suggest that, for entrapment neuropathies, ultrasound is a sensitive diagnostic tool which correlates with electrodiagnostic findings: in carpal tunnel syndrome, nerve CSA and nerve hypoechogenicity were always normal in minimal or mild cases and always altered in moderate to extreme cases. Also, the presence of epineurial hyperechogenicity indicated a more advanced degree of severity, and could represent a good indicator of epineurial fibrosis and need for surgical neurolysis. In cubital tunnel syndrome, ultrasound was even more sensitive, nerve CSA and nerve echogenicity being altered also in mild cases.
From a surgical point of view, ultrasound facilitated surgical approach by showing anatomical or dynamic alterations such as a bifid median nerve or nerve dislocation that would have not be identified preoperatively otherwise. If it is true that these alterations would not have altered the surgical plan, it is also true that this thorough preoperative evaluation allowed for a better discussion with the patient and for faster and easier surgery, at the expense of only the small amount of extra time required for the preoperative ultrasound evaluation.

Our findings are supported by the data in the literature, which show an emerging diagnostic role for ultrasound in entrapment neuropathies. Simon et al. ${ }^{29}$ reported that changes in the CSA and echogenicity correlate with the severity of the lesion. Filippou et al., ${ }^{9}$ Yoon et al., ${ }^{32}$ and Simon et al. ${ }^{29}$ found high-resolution ultrasonography more useful than electrodiagnostic tests for ulnar neuropathy; also, Beekmann et al. ${ }^{2}$ showed that the sensitivity of electrodiagnostic tests can be increased from $78 \%$ to $98 \%$ by adding ultrasonography, while Pardal-Fernandez ${ }^{27}$ even proposed substituting ultrasonography for EMG and velocities conduction studies as a first-line examination in selected cases. Cesmebasi et al. ${ }^{5}$ also reported on the usefulness of dynamic ultrasound in identification of a palmaris profundus tendon, a feature that, determining a dynamic compression, cannot be shown by MRI.

\section{Posttraumatic and Postsurgical Neuropathies}

In posttraumatic or postsurgical cases, the main contribution of ultrasound was visualization of nerve continu- 


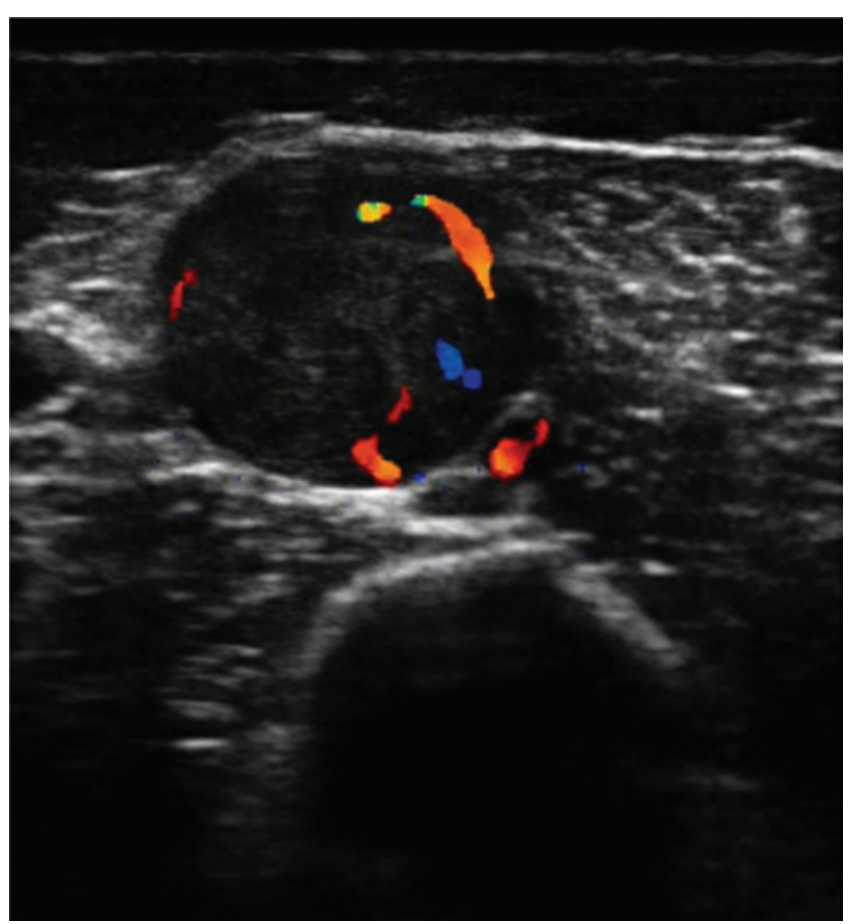

FIG. 10. Same schwannoma as in Figs. 8 and 9 in an axial and color Doppler ultrasound scan, which shows tumor neovascularization. The feeding artery, the plexiform veins, and eventual hypervascularity can be visualized with this technique. Figure is available in color online only.

ity. It allowed not only the identification of an amputation neuroma of larger nerves (i.e. median or ulnar) but also of small terminal branches (common palmar and proper palmar digital nerves) or neuromas-in-continuity, distinguishing interrupted fascicles from those in continuity.

Ultrasound was particularly useful in cases with atypical clinical or neurophysiological characteristics, as already reported by Padua et al., ${ }^{24}$ and in all cases in which no neural response could be evoked. Also, it was useful in identifying multifocal damage or uncommon sites of injury, which cannot be achieved by electrodiagnostic testing alone. As reported by other authors, ${ }^{8,14,21}$ ultrasound facilitated surgical planning in patients with neuromas, foreign bodies, and postfracture complications, allowing for targeted surgery and avoiding wide accesses for exploration because of accurate localization and estimation of nerve damage.

\section{Tumors}

The contributive role of ultrasound was more significant for tumors, in which it was diagnostic in $100 \%$ of cases, all of which had negative results on routine electrodiagnostic examinations. These findings were in accordance with the literature: Gruber et al. ${ }^{16}$ proposed ultrasound as the first imaging technique for a superficial mass of suspected nervous origin; Simon et al. ${ }^{28}$ reported a close correlation between ultrasound and MR tractography findings and considered ultrasound a valuable preoperative investigation for evaluating the risk of iatrogenic injury and for planning the optimal surgical strategy.

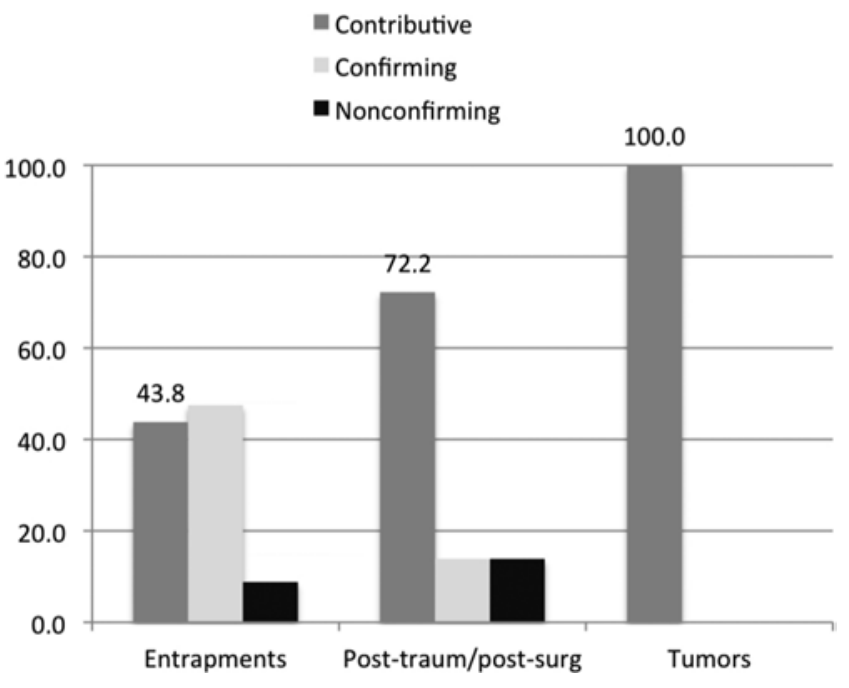

FIG. 11. Role of ultrasound with respect to neurophysiological examination in diagnosis and surgical planning in the 3 different groups of peripheral neuropathies. post-traum/post-surg $=$ posttraumatic/postsurgical.

In our study, we observed a higher contributive role of ultrasound than was reported in the study by Padua et al..$^{25}$ $(53.8 \%$ vs $42.3 \%)$ and a lower rate of negative (not confirming) role (10.1\% vs $17.7 \%$ ), which could be explained by a selection bias of our study population, as all patients in our series were candidate to surgery.

Based on the results of our study, we advocate the routine use of ultrasound nerve imaging in the evaluation of surgical candidates and support it as first-line imaging technique for the study of peripheral neuropathies, as a valuable complement to clinical and electrodiagnostic examination. Electrodiagnostic testing still represents the standard diagnostic tool for peripheral neuropathies as it provides unique information on the type of fibers involved (sensory or motor) and on the pathophysiology of the lesion (demyelination and/or axonal damage), but it may be uncomfortable for patients. We believe that ultrasound should be considered as a useful complement-not an alternative-to electrodiagnostic testing, although, as the diagnostic accuracy of imaging techniques increases, some authors foresee that high-resolution imaging could theoretically replace the electrodiagnostic tests in the diagnosis of some neuropathies. ${ }^{20,27}$

Ultrasound yielded negative results in cases of myeloradiculopathy (cervical or lumbar), in which motor/somatosensory evoked potential testing and MRI allowed final diagnosis. Thus, if proximal nerve impairment is suspected to be the main cause or to contribute to the clinical picture, ultrasound should not be recommended as the first-line imaging technique, and MRI shows a higher diagnostic value.

Our study has limitations: being a retrospective study, it is less reliable than a prospective study with regard to patient selection, and it did not include a control group. However, patient selection followed clearly defined inclusion and exclusion criteria based on our routine clinical protocol.

The study was not blinded, as the same clinician per- 
forming the electrodiagnostic tests performed also the ultrasound examination. This could introduce a bias in the evaluation of their correlation; however, the aim of the study was not to evaluate the role of ultrasound alone but its role as a complementary evaluation in a practical clinical setting, where all diagnostic tests are performed during the same medical examination.

Our patient population was very heterogeneous, thus the numbers for each single nerve and type and level of lesion are limited; statistical analysis was performed only on a part of the data, as some subgroups (e.g., tumors) included only a small number of patients. However, this clinical series reflects the variability encountered in clinical practice and shows how nerve ultrasound contributes to the daily evaluation and management of focal peripheral nerve injuries. The influence of diabetes and other predisposing factors on nerve ultrasound characteristics was not evaluated. The duration of symptoms in the posttraumatic or postsurgical groups showed a wide range (2-72 months), and differences in nerve ultrasound findings were not evaluated with respect to this variable. Only a few patients in this subgroup received an early evaluation, and the role of ultrasound in this subset of patients was not evaluated, but could prove even more crucial: it is often difficult, with clinical and neurophysiological examination, to determine the type of lesion and the timing of surgery in the early phases, while ultrasound could allow early differentiation of neurotmetic from axonotmetic injuries, avoiding unnecessary delay in treatment.

\section{Conclusions}

Ultrasound is a powerful, noninvasive tool for examination of peripheral nerve injuries, which can guide diagnosis and surgical strategy of focal peripheral nerve injuries. It allows direct visualization of the cause and extent of nerve lesions and finds its place between electrodiagnostic tests and exploratory surgery. It can be used to complement a doubtful electrodiagnostic test, providing invaluable information, such as the presence and extent of a mass, scar compression, or neuromas. We recommend it as a complement to routine clinical and neurophysiological routine evaluation and as a first-line imaging modality for masses of suspected nerve origin.

\section{References}

1. Alaqeel A, Alshomer F: High resolution ultrasound in the evaluation and management of traumatic peripheral nerve injuries: review of the literature. Oman Med J 29:314-319, 2014

2. Beekman R, Van Der Plas JP, Uitdehaag BM, Schellens RL, Visser LH: Clinical, electrodiagnostic, and sonographic studies in ulnar neuropathy at the elbow. Muscle Nerve 30:202208, 2004

3. Caliandro P, Foschini M, Pazzaglia C, La Torre G, Aprile I, Granata G, et al: IN-RATIO: a new test to increase diagnostic sensitivity in ulnar nerve entrapment at elbow. Clin Neurophysiol 119:1600-1606, 2008

4. Cartwright MS, Chloros GD, Walker FO, Wiesler ER, Campbell WW: Diagnostic ultrasound for nerve transection. Muscle Nerve 35:796-799, 2007

5. Cesmebasi A, Spinner RJ, Smith J, Martinoli C: Dynamic ultrasonography can demonstrate the mechanism of the palmaris profundus in carpal tunnel syndrome. Clin Anat 28:428-430, 2015

6. Daube JR: AAEM minimonograph \#11: Needle examination in clinical electromyography. Muscle Nerve 14:685-700, 1991

7. Di Pasquale A, Morino S, Loreti S, Bucci E, Vanacore N, Antonini G: Peripheral nerve ultrasound changes in CIDP and correlations with nerve conduction velocity. Neurology 84:803-809, 2015

8. Erra C, Granata G, Liotta G, Podnar S, Giannini M, Kushlaf $\mathrm{H}$, et al: Ultrasound diagnosis of bony nerve entrapment: case series and literature review. Muscle Nerve 48:445-450, 2013

9. Filippou G, Mondelli M, Greco G, Bertoldi I, Frediani B, Galeazzi M, et al: Ulnar neuropathy at the elbow: how frequent is the idiopathic form? An ultrasonographic study in a cohort of patients. Clin Exp Rheumatol 28:63-67, 2010

10. Filler AG, Howe FA, Hayes CE, Kliot M, Winn HR, Bell BA, et al: Magnetic resonance neurography. Lancet 341:659-661, 1993

11. Gagliardo A, Avarino C, Giaimi G, Di Matteo D, Midiri M, Gagliardo C: Ultrasound combined with clinical neurophysiology in peripheral nerve pathologies: when it is worth? Preliminary data in 50 outpatients. Clin Neurophysiol 124:e189, 2013 (Abstract)

12. Gagliardo A, Avarino C, Giaimi G, Di Matteo D, Midiri M, Gagliardo C: Emerging role of Ultrasound imaging associated to Clinical Neurophysiology as an advanced diagnostics of peripheral nerves pathologies. A Sicilian experience. Neuroradiology 55:S114, 2013 (Abstract)

13. Gagliardo A, Toia F, Maggì F, Mariolo AV, Cillino M, Moschella F: Clinical neurophysiology and imaging of nerve injuries: preoperative diagnostic work-up and post-operative monitoring. Plast Aesthet Res 2:149-155, 2015

14. Gofeld M, Bristow SJ, Chiu S, Kliot M: Preoperative ultrasound-guided mapping of peripheral nerves. J Neurosurg 119:709-713, 2013

15. Grimm A, Heiling B, Schumacher U, Witte OW, Axer H: Ultrasound differentiation of axonal and demyelinating neuropathies. Muscle Nerve 50:976-983, 2014

16. Gruber H, Glodny B, Bendix N, Tzankov A, Peer S: Highresolution ultrasound of peripheral neurogenic tumors. Eur Radiol 17:2880-2888, 2007

17. Howe BM, Spinner RJ, Felmlee JP, Amrami KK: High-resolution imaging of upper limb neuropathies. Semin Musculoskelet Radiol 19:160-167, 2015

18. Howe FA, Filler AG, Bell BA, Griffiths JR: Magnetic resonance neurography. Magn Reson Med 28:328-338, 1992

19. Iannicelli E, Almberger M, Chianta GA, Salvini V, Rossi G, Monacelli G, et al: High resolution ultrasonography in the diagnosis of the carpal tunnel syndrome. Radiol Med (Torino) 110:623-629, 2005

20. Jarvik JG, Comstock BA, Heagerty PJ, Haynor DR, FultonKehoe D, Kliot M, et al: Magnetic resonance imaging compared with electrodiagnostic studies in patients with suspected carpal tunnel syndrome: predicting symptoms, function, and surgical benefit at 1 year. J Neurosurg 108:541-550, 2008

21. Kara M, Ekiz T, Öztürk GT, Onat ŞŞ, Özçakar L: Heterotopic ossification and peripheral nerve entrapment: ultrasound is a must-use imaging modality. Pain Med 16:1643-1644, 2015 (Letter)

22. Moran L, Perez M, Esteban A, Bellon J, Arranz B, del Cerro M: Sonographic measurement of cross-sectional area of the median nerve in the diagnosis of carpal tunnel syndrome: correlation with nerve conduction studies. J Clin Ultrasound 37:125-131, 2009

23. Naranjo A, Ojeda S, Mendoza D, Francisco F, Quevedo JC, Erausquin C: What is the diagnostic value of ultrasonography compared to physical evaluation in patients with idiopathic 
carpal tunnel syndrome? Clin Exp Rheumatol 25:853-859, 2007

24. Padua L, Aprile I, Pazzaglia C, Frasca G, Caliandro P, Tonali $\mathrm{P}$, et al: Contribution of ultrasound in a neurophysiological lab in diagnosing nerve impairment: A one-year systematic assessment. Clin Neurophysiol 118:1410-1416, 2007

25. Padua L, Liotta G, Di Pasquale A, Granata G, Pazzaglia C, Caliandro P, et al: Contribution of ultrasound in the assessment of nerve diseases. Eur J Neurol 19:47-54, 2012

26. Padua L, LoMonaco M, Gregori B, Valente EM, Padua R, Tonali P: Neurophysiological classification and sensitivity in 500 carpal tunnel syndrome hands. Acta Neurol Scand 96:211-217, 1997

27. Pardal-Fernandez JM: [Carpal tunnel syndrome. The contribution of ultrasonography.] Rev Neurol 59:459-469, 2014 (Sp)

28. Simon NG, Cage T, Narvid J, Noss R, Chin C, Kliot M: Highresolution ultrasonography and diffusion tensor tractography map normal nerve fascicles in relation to schwannoma tissue prior to resection. J Neurosurg 120:1113-1117, 2014

29. Simon NG, Ralph JW, Poncelet AN, Engstrom JW, Chin C, Kliot M: A comparison of ultrasonographic and electrophysiologic 'inching' in ulnar neuropathy at the elbow. Clin Neurophysiol 126:391-398, 2015

30. Solbiati L, De Pra L, Ierace T, Bellotti E, Derchi LE: Highresolution sonography of the recurrent laryngeal nerve: anatomic and pathologic considerations. AJR Am J Roentgenol 145:989-993, 1985

31. Stevens JC: AAEM minimonograph \#26: the electrodiagnosis of carpal tunnel syndrome. Muscle Nerve 20:1477-1486, 1997

32. Yoon JS, Walker FO, Cartwright MS: Ulnar neuropathy with normal electrodiagnosis and abnormal nerve ultrasound. Arch Phys Med Rehabil 91:318-320, 2010
33. Zaidman CM, Seelig MJ, Baker JC, Mackinnon SE, Pestronk A: Detection of peripheral nerve pathology: comparison of ultrasound and MRI. Neurology 80:1634-1640, 2013

\section{Disclosures}

The authors report no conflict of interest concerning the materials or methods used in this study or the findings specified in this paper.

\section{Author Contributions}

Conception and design: A Gagliardo, Toia, Cordova. Acquisition of data: A Gagliardo, D'Arpa, C Gagliardo. Analysis and interpretation of data: A Gagliardo, Toia, C Gagliardo. Drafting the article: A Gagliardo, Toia. Critically revising the article: A Gagliardo, D'Arpa, Cordova. Reviewed submitted version of manuscript: A Gagliardo, Toia. Approved the final version of the manuscript on behalf of all authors: A Gagliardo. Statistical analysis: A Gagliardo. Administrative/technical/material support: G Gagliardo. Study supervision: A Gagliardo, G Gagliardo, Cordova.

\section{Supplemental Information Videos \\ Video 1. https://vimeo.com/140429696.}

\section{Correspondence}

Andrea Gagliardo, "Clinical Course" Neurophysiology Unit, via A. De Gasperi, 81, Palermo 90146, Italy. email: andrigl@ gmail. com. 